\title{
Non-Destructive Isosurface 3-D Modeling by Scanning Electron Tomography
}

\author{
T.S. Yeoh,* N. A. Ives
}

The Aerospace Corporation, 2350 E. El Segundo B1. El Segundo, CA 90245-4691

Transmission electron tomography and focused ion beam nanotomography are both destructive techniques that have been used to image and model nanostructures [1-3]. However, there are instances in which structural information such as orientation, length and surface morphology must be obtained without damaging the sample. In this case, a different approach is taken. A scanning electron beam tilt series spanning 120 degrees has been used to reconstruct isosurface three-dimensional models of self-assembled, freestanding semiconductor nanowires and nanohelices as shown in Fig 1. This was accomplished by applying a special case in computer-aided tomography that assumes complete transmission or complete absorption.

By utilizing this special case, isosurfaces were generated from the tomographic computations. The resulting model as shown in Fig 2 gave not only information on the orientation and thickness, but also revealed important features from within the structure such as the number of curls and the orientation of the curls with respect to the structure.

Additionally, the tomographic reconstruction provided details of the surface underneath the freestanding structures, which is not normally achievable by standard scanning electron microscope image stereograms. The tomographic computation of isosurfaces using the scanning electron microscope provides a nondestructive method for accurately determining physical attributes on the micron to nanometer scales without having to destroy or modify the sample of interest.

[1] Z. H. Levine, A. R. Kalukin, M. Kuhn, S. P. Frigo, I. McNulty, C. C. Retsch, Y. Wang, U. Arp, T. B. Lucatorto, B. D. Ravel, and C. Tarrio, "Tomography of integrated circuit interconnect with an electromigration void," Journal of Applied Physics, vol. 87, pp. 4483-4488, 2000.

[2] D. N. Dunn, G. J. Shiflet, and R. Hull, "Quantitative three-dimensional reconstruction of geometrically complex structures with nanoscale resolution," Review of Scientific Instruments, vol. 73, pp. 330-334, 2002.

[3] E. Lee, R. Williams, G. B. Viswanathan, R. Banerjee, and H. L. Fraser, "3D Materials Characterization using Dual-Beam FIB/SEM Techniques," Microsc Microanal, vol. 10, pp. 11281129, 2004.

[4] This work was supported under The Aerospace Corporation's Independent Research and Development Program. 


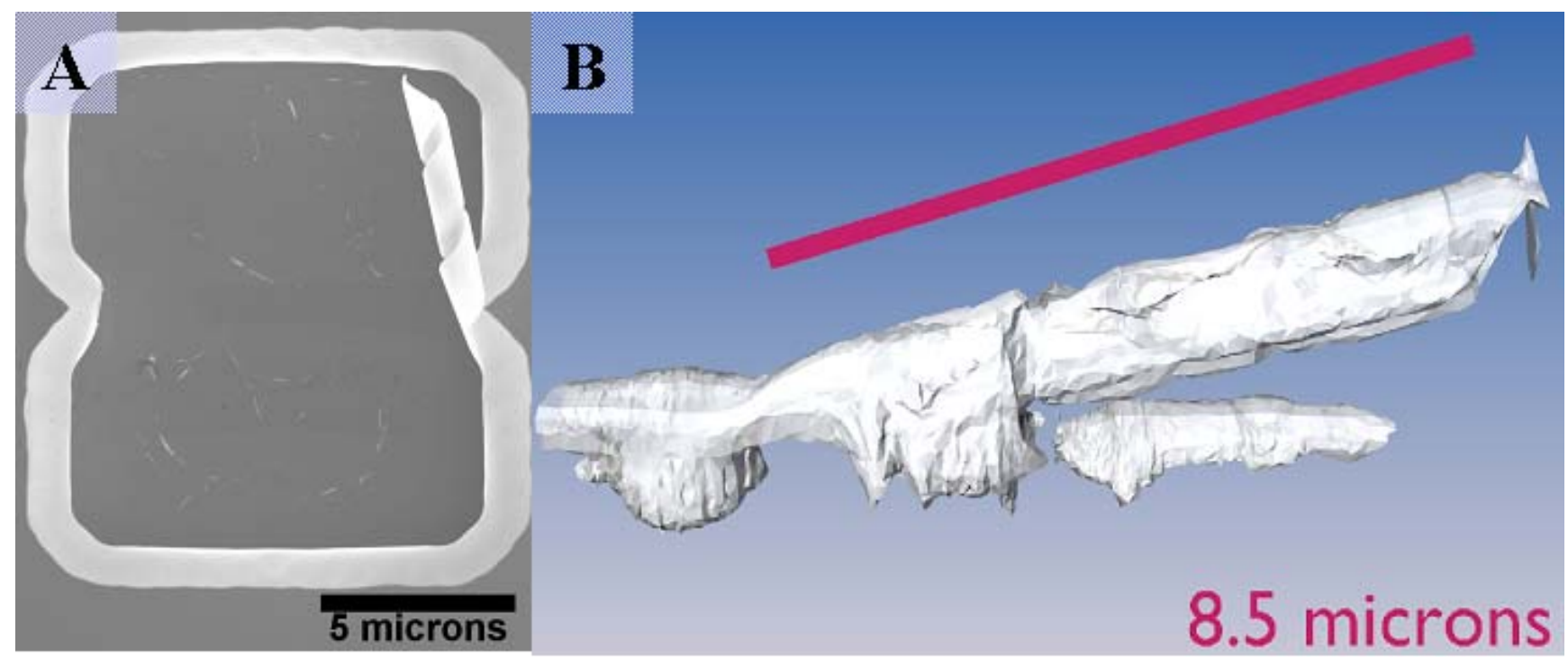

Fig 1. SEM micrograph showing one of the 120 tilt series images of a nanoscroll self-assembled structure (A). The tilt series were aligned and the resulting sinogram and isosurface (B) show the structure in three dimensions.

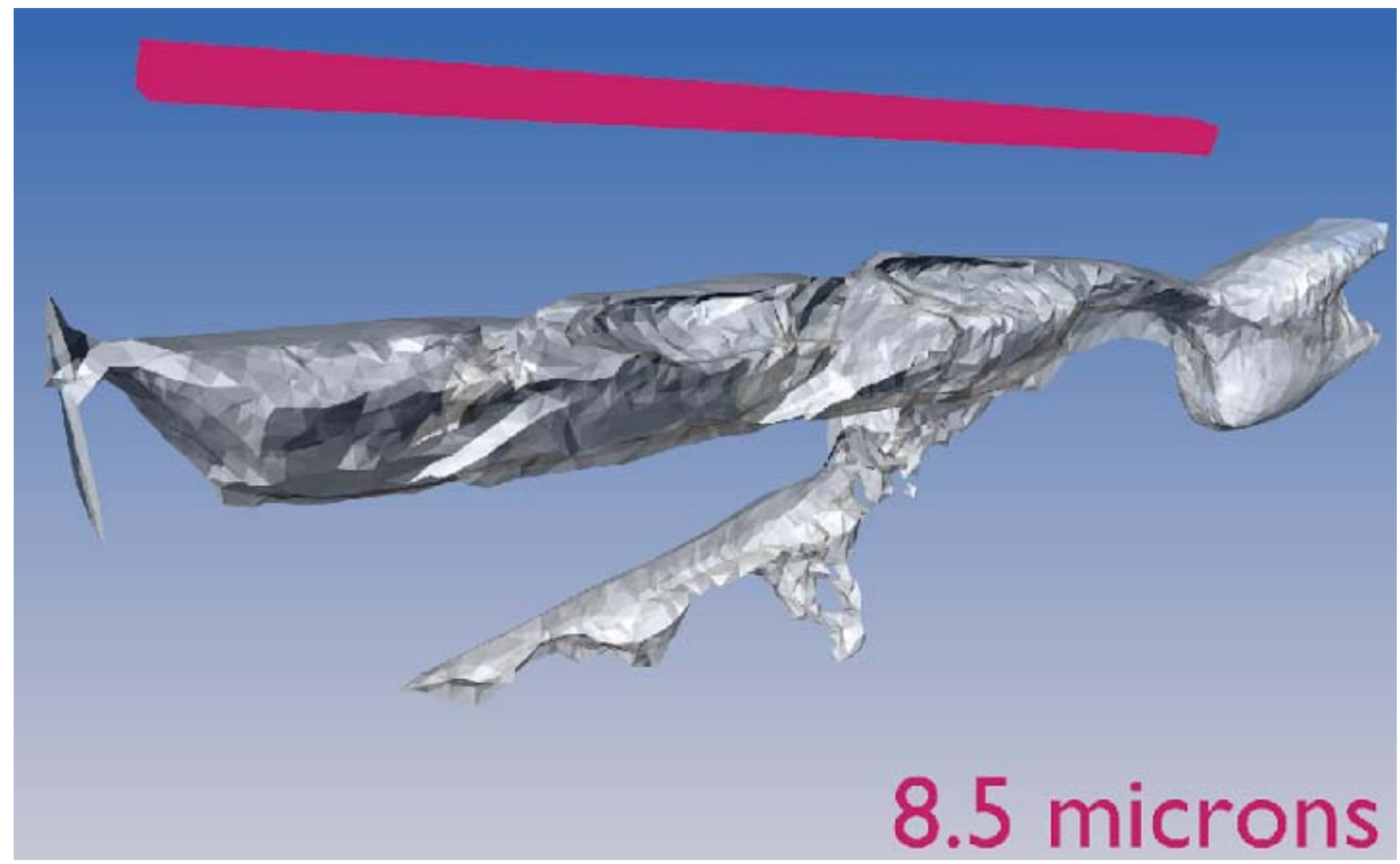

Fig 2. Once in an isosurface format, the structure can be rotated and examined from various directions. In this direction, the individual scrolling elements can be observed. Accurate lengths and thicknesses can be derived using this non-destructive technique. 